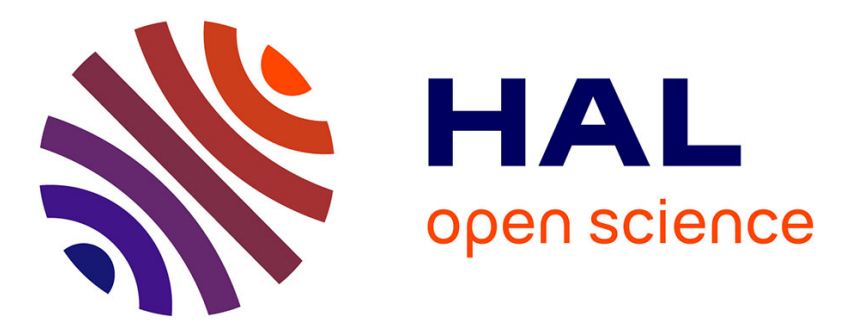

\title{
Anisotropic exciton states in GaAs/AlAs superlattices in zero and non-zero magnetic field
}

C. Gourdon, D. Rodichev, P. Lavallard, G. Bacquet, R. Planel

\section{To cite this version:}

C. Gourdon, D. Rodichev, P. Lavallard, G. Bacquet, R. Planel. Anisotropic exciton states in GaAs/AlAs superlattices in zero and non-zero magnetic field. Journal de Physique IV Proceedings, 1993, 03 (C5), pp.C5-183-C5-186. 10.1051/jp4:1993534 . jpa-00251620

\section{HAL Id: jpa-00251620 https://hal.science/jpa-00251620}

Submitted on 1 Jan 1993

HAL is a multi-disciplinary open access archive for the deposit and dissemination of scientific research documents, whether they are published or not. The documents may come from teaching and research institutions in France or abroad, or from public or private research centers.
L'archive ouverte pluridisciplinaire HAL, est destinée au dépôt et à la diffusion de documents scientifiques de niveau recherche, publiés ou non, émanant des établissements d'enseignement et de recherche français ou étrangers, des laboratoires publics ou privés. 


\title{
Anisotropic exciton states in Gras/AlAs superlattices in zero and non-zero magnetic field
}

\author{
C. GOURDON, D.Yu. RODICHEV ${ }^{(1)}$, P. LAVALLARD, G. BACQUET* and R. PLANEL** \\ Groupe de Physique des Solides, URA 17 du CNRS, Universités Paris 6 et 7, 2 place Jussieu, 75251 Paris \\ cedex 05, France \\ * Laboratoire de Physique des Solides, URA 74 du CNRS, INSA, avenue de Rangueil, 31077 Toulouse \\ cedex, France \\ ${ }^{* *}$ L2M-UP CNRS 20, 196 avenue H. Ravera, 92220 Bagneux, France
}

\begin{abstract}
In short period pseudodirect GaAs/AlAs superlattices, the two radiative heavy exciton states exhibit a very small anisotropic splitting due to their coupling with light exciton states. They are dipole-active along the [110] and [110] directions in the layer plane. The splitting energy (a few $\mu \mathrm{eV}$ ) is obtained from the period of the quantum beats between the two sublevels observed during the time decay of photoluminescence under excitation with light linearly polarized along [100] or circularly polarized. We report here on the variation of the quantum beats in a magnetic field. In a longitudinal field (parallel to the wave vector of light), we measure the splitting between the radiative states and obtain the hole longitudinal g-factor. In a transverse field, we observe two periods for the quantum beats. One corresponds to the splitting between the radiative states and the other to the splitting between the radiative states and the non-radiative ones which become optically allowed in a transverse field. We also discuss the influence of the coupling to light excitons in a transverse field on the hole transverse g-factor.
\end{abstract}

\section{INTRODUCTION}

In the recent years, the fine structure of the heavy exciton (HE) state in pseudodirect type II GaAs/AlAs superlattices (SL) has been investigated by several methods $(1,2,3)$. In an ideal SL, the HE state consists of four levels built from the heavy hole states with projection of the total angular momentum along the [001] growth axis ( $z$ axis) $J_{z}= \pm 3 / 2$ and the electron states with spin $S_{z}= \pm 1 / 2$. Owing to the electron-hole exchange interaction, the exciton state is split into a radiative doublet and two non-radiative states. However, it was found experimentally that the degeneracy of the radiative doublet is lifted and that the two sublevels are dipole-active along the [110] and [110] directions in the layer plane. As a matter of fact, the overlap of the envelope functions of the electron (mainly confined in AlAs layers) and of the hole (mainly confined in GaAs layers) is maximum at the interfaces. The lifting of the degeneracy of the radiative exciton states is explained by the lower symmetry of the interface and by the localization of the exciton wave function mainly on one interface owing to interface roughness. The splitting energy $\Delta$, whose value is of the order of a few $\mu \mathrm{eV}$, was experimentally deduced from the period of the quantum beats observed during photoluminescence (PL) time decay when the [110] and[110] sublevels are coherently excited by a laser pulse linearly polarized along [100] or [010], or circularly polarized $(3,4) . \Delta$ was calculated by taking into account the coupling of heavy and light exciton (LE) states. Using a perturbation theory, we found that the combined effect of the electron-hole exchange interaction and a perturbation $Z$ having the symmetry of an $\varepsilon_{\mathrm{xy}}$ strain leads to a splitting energy $\Delta$ equal to $4 \mathrm{EZ} /\left(\sqrt{3} \Delta_{\mathrm{lh}}\right)$ where $\mathrm{E}$ is the electron-hole exchange energy and $\Delta_{\mathrm{lh}}$ the energy separation between heavy and light excitons $\left({ }^{4}\right)$. The $\varepsilon_{\mathrm{xy}}$ strain was assumed to exist only at the interfaces and was attributed to the fact that As- $\mathrm{Al}$ and As-Ga bonds lie in the (110) and (110) planes on each side of the interfaces. The fit with experimental results for various SLs gave $Z=14.5 \mathrm{meV}$.

(1) Permanent address: Physics Department, Moscow State University, Lenin Hills, 117234 Moscow, Russia 
Another explanation was proposed by Aleiner and Ivchenko 5 . The splitting arises from an intrinsic effect: the mixing of heavy and light hole states at the interface due to the lower symmetry. They use extended boundary conditions for the hole envelope functions with a mixing coefficient $t_{\mathrm{l}}$ which is found equal to 1.4 from a fit with experimental results.

It was shown experimentally that, in some samples, there are two classes of excitons with opposite signs of the anisotropic exchange splitting $\Delta(1,3,4)$. In both models, these two classes are related to GaAs/AlAs and AlAs/GaAs interfaces.

We present here results on quantum beats between excitonic sublevels in a longitudinal or a transverse magnetic field. We show for the first time the appearance of two different periods for the quantum beats observed in a transverse field. The experimental results were obtained with two GaAs/AlAs SL samples of composition $18 \AA / 12 \AA$ and $22 \AA / 11.5 \AA$ grown by molecular beam epitaxy. The samples were immersed in superfluid liquid helium. PL was quasi-resonantly excited, at nearly normal incidence, with circularly or linearly polarized laser pulses of about $10 \mathrm{ps}$ from a tunable dye laser pumped by an $\mathrm{Ar}^{+}$- ion laser. In both configurations (longitudinal or transverse), the magnetic field was created by superconducting coils placed in the cryostat. The polarized PL was analysed by a Babinet-Soleil compensator followed by a polarizer and a monochromator and detected with either a photomultiplier or a synchroscan streak camera with a time resolution of about 30 ps.

\section{EXPERIMENTAL RESULTS AND DISCUSSION}

The exciton hamiltonian in a magnetic field is written as in ref.( $\left.{ }^{1}\right)$ :

$\mathrm{H}_{\mathrm{B}}=\mu_{\mathrm{B}} \sum_{\mathrm{i}=\mathrm{x}, \mathrm{y}, \mathrm{z}, \mathrm{i}} \mathrm{g}_{\mathrm{e}, \mathrm{i}} \mathrm{B}_{\mathrm{i}}-2 \mu_{\mathrm{B}} \sum_{\mathrm{i}=\mathrm{x}, \mathrm{y}, \mathrm{z}}\left(\kappa J_{\mathrm{h}, \mathrm{i}}+\mathrm{qJ}_{\mathrm{h}, \mathrm{i}}^{3}\right)_{\mathrm{i}}$

where $\mu_{\mathrm{B}}$ is the Bohr magneton and $\mathrm{g}_{\mathrm{e}}$ the electron g-factor. Neglecting the coupling with light excitons, this expression can be written in the form $\left(^{1}\right)$ :

$H_{B}=\mu_{B} \sum_{i=x, y, z}\left(g_{e, i} S_{e, i}-g_{h, i} S_{h, i}\right) B_{i}$

where $S_{h}$ is an effective spin for the heavy hole $\left(J_{h, z}=+3 / 2(-3 / 2)\right.$ corresponding to $S_{h, z}=+1 / 2(-1 / 2)$, respectively) and $\mathrm{g}_{\mathrm{h}}$ the $\mathrm{g}$-factor of the hole.

\subsection{Longitudinal magnetic field}

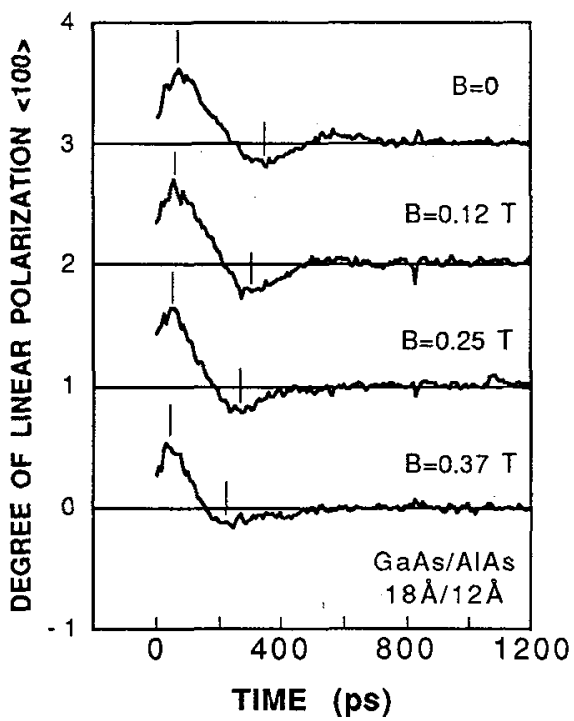

The field is colinear with the [001] growth axis of the sample. The two radiative states are coupled to each other by the magnetic field $B$. Their splitting energy varies as $\left.\Delta_{B}=\sqrt{\Delta^{2}+\left(\left(g_{e z}+g_{h z}\right) \mu_{B} B\right.}\right)^{2}$. A coherent superposition of these $\langle 110\rangle$ dipole-active states is excited by a laser pulse polarized along a $<100>$ direction in the layer plane. The PL intensity I is detected for polarizations parallel (II) and perpendicular $(\perp)$ to the excitation. Owing the energy difference $\Delta_{B}$ between the two $\left.<110\right\rangle$ states, $I_{\| l}$ and $I_{\perp}$ oscillate during their time decay with opposite phases and with a period $T$ equal to $2 \pi \mathrm{h} / \Delta_{\mathrm{B}}$. The $\langle 100\rangle$ degree of linear polarization DLP $<100>$, defined as $\left(I_{\| l}-I_{\perp}\right) /\left(I_{\| l}+I_{\perp}\right)$ is shown as a function of the magnetic field in Fig.1. One clearly observes the decrease of the period of the quantum beats, i.e. the increase of the splitting, with the increase of the field. The value of the electron g-factor being wellknown $\left(\mathrm{gez}_{\mathrm{e}}=1.905\left(^{6}\right)\right.$ ), we determine the hole longitudinal $\mathrm{g}$-factor $\mathrm{ghz}_{\mathrm{h}}=-2.2 /-2.3$ from a fit of the splitting as a function of the field. This value is in good agreement with those determined in ref. $\left({ }^{1}\right)$ by optically detected magnetic resonance for pseudodirect SLs.

Fig.1 Quantum beats in a longitudinal field for a $18 \AA / 212 \AA$ GaAs/AlAs SL. The zero line is shifted by 1 for each curve.

With the increase of the field the two radiative exciton states, initially of linear symmetry along the $<110\rangle$ axes recover a circular symmetry. In stationary measurements, as already shown in ref. $\left({ }^{7}\right)$, one observes an increase of the degree of circular polarization (DCP) up to its saturation value, together with a decrease of the DLP measured with $<110>$ polarized excitation. The DCP 
and $D_{L P}<110>$ are calculated in the frame of the density-matrix formalism as described in ref. $\left({ }^{4,7}\right)$. We obtain a good fit with the experimental DCP and DLP $<110>$ using the $g_{h z}$ value determined above, a lifetime $\tau_{0}=28 \mathrm{~ns}$ and a spin relaxation time $\tau_{\mathrm{S}}=43 \mathrm{~ns}$ obtained from the time-resolved $\mathrm{DLP}<110>$ as explained in ref. $\left({ }^{4}\right)$.

\subsection{Transverse magnetic field}

The field is directed along one of the $\langle 110\rangle$ directions, let us say along [110] for calculation. In the first approximation, the coupling of heavy and light excitons through the [110] magnetic field is neglected, i.e. we use the Hamiltonian given in equation (2). The exciton eigenstates at zero field are taken as $|\mathrm{r} 1\rangle=\frac{1}{\sqrt{2}}[\mathrm{i}|-1 / 2,3 / 2\rangle+|1 / 2,-3 / 2\rangle]$ and $|\mathrm{r} 2\rangle=\frac{1}{\sqrt{2}}[|-1 / 2,3 / 2\rangle+\mathrm{i}|1 / 2,-3 / 2\rangle]$ for the radiative ones (with eigenenergies $\Delta / 2$ and $-\Delta / 2$ for $|r 1\rangle$ and $|r 2\rangle$ respectively) and $\ln r 1\rangle=\frac{1}{\sqrt{2}}[|1 / 2,3 / 2\rangle+|-1 / 2,-3 / 2\rangle]$ and $|\mathrm{nr} 2\rangle=\frac{1}{\sqrt{2}}[|1 / 2,3 / 2\rangle-|-1 / 2,-3 / 2\rangle]$ for the non-radiative ones (with eigenenergies $-E+\delta / 2$ and $-E-\delta / 2$ for $\ln 1\rangle$ and $\ln r 2\rangle$, respectively). The total Hamiltonian (anisotropic exchange interaction + magnetic field) has a simple expression in this basis with two non-diagonal matrix elements: $\langle n r 1 \mid r 1\rangle=\left(g_{e \perp}-g_{h \perp}\right) \beta(1+i)$ and $\langle n r 2 \mid r 2\rangle=\left(g_{e}+g_{h \perp}\right) \beta(1-i)$, where $\beta=\frac{\mu_{B} B}{2 \sqrt{2}}$, $g_{h \perp}=3 q$, $g_{e \perp}$ and $g_{h} \perp$ being the electron and hole transverse g-factors, respectively. $g_{h} \perp$ was found to be very small $(<0.01)$ for the samples studied in ref.( $\left.{ }^{1}\right)$.

Owing to the anisotropic exchange coupling between heavy and light excitons, the SL lowest excitonic eigenstates are in fact a superposition of $\mathrm{HE}$ eigenstates (as written above) and LE eigenstates with a coefficient of the order of $Z / \Delta_{\operatorname{lh}}\left({ }^{8}\right)$. Since the value of $Z$ was experimentally found to be $14.5 \mathrm{meV}\left({ }^{4}\right)$, it may be questionable to neglect the coupling of heavy and light excitons by the magnetic field when $\mathrm{Z} / \Delta_{\mathrm{lh}}$ is not very small, as it is the case for our samples. Therefore we calculated the excitonic eigenenergies using the Hamiltonian (1) which couples the radiative $\mathrm{HE}$ states not only to the non-radiative HE states but also to the non-radiative LE states. Neglecting terms in $\left(\mathrm{Z} / \Delta_{\mathrm{lh}}\right)^{2}$ and $\mathrm{E} / \Delta_{\mathrm{lh}}$ and neglecting $\mathrm{E}$ with respect to $\mathrm{Z}$ in the calculation of the non-diagonal matrix elements, we calculate the Hamiltonian on the basis of SL excitonic states $\left.\ln r^{\prime} 1\right\rangle,\left|r^{\prime} 1\right\rangle,\left|n r^{\prime} 2\right\rangle,\left|r^{\prime} 2\right\rangle$ in which the LE character has been taken into account. We find that $\left.\left\langle n r^{\prime} 1 \mid r^{\prime} 1\right\rangle=g_{e \perp}-3 q+\frac{4 \sqrt{3} Z}{\Delta_{l h}}\left(k+\frac{7 q}{4}\right)\right) \beta(1+i)$ and $\left\langle n r^{\prime} 2 \mid r^{\prime} 2\right\rangle=\left(g_{e}+3 q\right) \beta(1-i)$. This is equivalent to an anisotropic hole transverse g-factor whose value along the direction of the field depends on $\mathrm{Z} / \Delta_{\mathrm{lh}}$. The same calculation in the case of a longitudinal field results in changes of the matrix elements by terms in $\left(\mathrm{Z} / \Delta_{\mathrm{lh}}\right)^{2}$ which are of the order of the experimental accuracy on the value of ghz. However, a numerical estimation of the effective transverse hole $\mathrm{g}$-factor for the SLs studied in ref.( ${ }^{1}$ ) gives values too large by a factor of 40. We also calculated the correction to the hole g-factor within the model of Aleiner and Ivchenko. Terms which are linear in the mixing coefficient $t_{l h}$ cancel out and only terms in $t_{l h}^{2}$ remain. The effective hole g-factor, which is the same for both non-diagonal matrix elements, is equal to $\left(3 q+4\left(\kappa+\frac{5 q}{2}\right) R\right) \cdot R$ is equal to the ratio $\int S^{2}(z) d z /\left[\int C^{2}(z) d z\right]$ where $S(z)$ and $C(z)$ and the envelope functions for the light hole and heavy hole states, respectively. $\mathrm{R}$ is proportional to $\mathrm{t}_{\mathrm{lh}}^{2}$. Numerical calculation of $g_{h} \perp$ gives a better agreement with experimental results for samples of ref.( $\left.{ }^{1}\right)$. Hence, we have to acknowledge that this is a strong argument in favor of the model of Aleiner and Ivchenko.

In transverse field, the variation of the stationary DCP or DLP with the field does not give much information about the parameters of the excitonic sublevels. The DLP ${ }_{110}$, slightly decreases with the field. The DLP $<100$, and the DCP show a small increase. On the contrary, the time-resolved DCP or the DLP $<100>$ give much insight into the behavior of the four sublevels. It is worth noting that the magnetic field couples the radiative states to the non-radiative ones but not to each other. Hence, as the field increases, the initially radiative states acquire a non-radiative part but the symmetry of their radiative part ( $\alpha 110\rangle$ excitonic dipole) is not modified. A coherent superposition of these levels can be excited either with a [100] polarized or with a $\sigma^{+}$circularly polarized light pulse. The time-resolved difference of intensities $\left(I_{\sigma+}-I_{\sigma-}\right)$ is shown in Fig.2 for different values of the magnetic field. One very clearly observes two periods for the quantum beats. The largest period corresponds to the first approximation to the small splitting energy $\Delta_{B}$ between $|r 1\rangle$ and $|r 2\rangle$ (Fig.3). The smallest period, in the range of 40 to $150 \mathrm{ps,} \mathrm{corresponds} \mathrm{to} \mathrm{the} \mathrm{large} \mathrm{splitting} \mathrm{energies} K \Omega_{+}$ 
and $\not \Omega$. between the states $|r 1\rangle$ and $|n r 2\rangle$ on the one hand and between $|r 2\rangle$ and $\ln 1\rangle$ on the other hand. At large field values, one has $\not h \Omega_{ \pm}=g_{e} \perp \mu_{B} B \pm(\Delta+\delta) / 2$. Since $\left(g_{e} \perp \mu_{B} B\right) »(\Delta+\delta) / 2$, one observes the mean splitting $h \Omega=\mathrm{g}_{\mathrm{e} \perp} \mu_{\mathrm{B}} \mathrm{B}$. This is in excellent agreement with our experimental result using $\mathrm{g}_{\mathrm{e} \perp}=1.97$ ( $^{\mathrm{l}}$ ). Oscillations are also observed on the sum $\left(I_{\sigma+}+I_{\sigma-}\right)$, corresponding to quantum beats between the coupled states $|r 1\rangle$ and $\ln r 1\rangle$, and $|r 2\rangle$ and $\ln 2\rangle$. It is worth noting that quantum beats can be observed between the radiative and non-radiative states because the latter ones acquire a radiative character as the field increases.

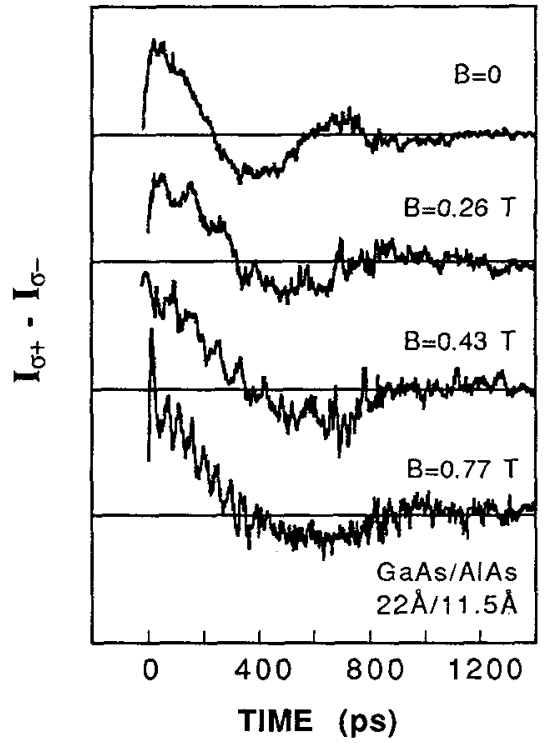

Fig. 2 Quantum beats in the difference $\left(I_{\sigma+}-I_{\sigma-}\right)$ in a transverse field directed along a $\langle 110\rangle$ axis. The zero is shifted for each curve.

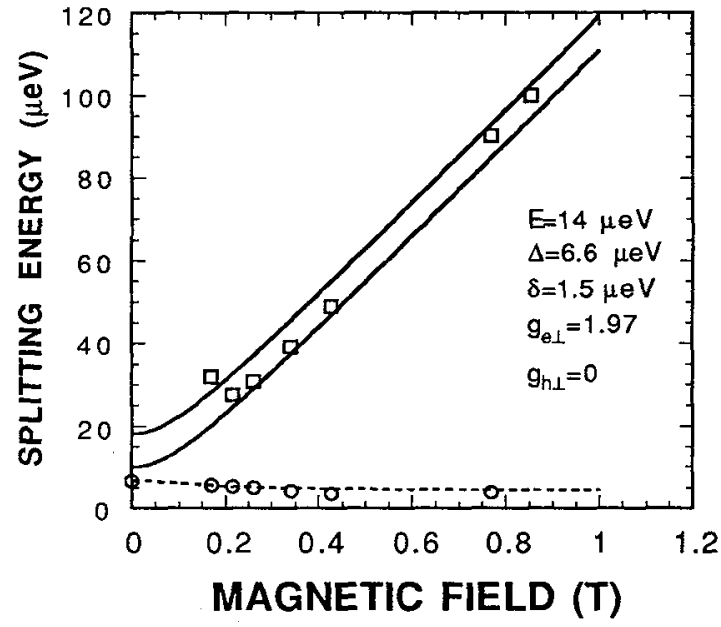

Fig. 3 The splitting energies deduced from the periods of the quantum beats in a transverse field are plotted as a function of the field. The full curves are the calculated energy differences between the states $|r|>$ and $\ln 2\rangle$ (upper curve) and between $|r 2\rangle$ and $\ln r \mid>$ (lower curve). The dashed curve is the calculated energy difference between $|r|\rangle$ and $|r 2\rangle$.

Using an enlarged energy scale, one can see that the splitting energy $\Delta_{\mathrm{B}}$ varies non monotonously with the field in the range 0.3-0.8 T. This may be attributed to $\ln 1>$ and $\ln r 2>$ states. Their splitting $\delta_{\mathrm{B}}$ may become large enough to be observed. Moreover, in the case of the existence of two classes of excitons, the splittings $\Delta_{B}$ and $\delta_{B}$ have different variations with the field for the two classes. Hence four splitting energies may become observable. Work is in progress to clarify this point.

As a conclusion, the observation of quantum beats in a magnetic field finds itself as a very powerful method to obtain information about the fine structure of the excitonic states. We determine various parameters of the excitonic sublevels such as splitting energies in the range of a few $\mu \mathrm{eV}$, hole longitudinal $\mathrm{g}$-factor, lifetime and spin relaxation times. In a transverse field, the non-radiative excitonic states become partly optically allowed. A detailed analysis of the large period of the quantum beats should give information about the splitting of these states at zero field. The splitting between these states and the $(B=0)$ radiative ones gives rise to quantum beats of small period corresponding to a splitting equal to $\mathrm{g}_{\mathrm{e}} \perp \mu_{\mathrm{B}} \mathrm{B}$.

[1] H.W. van Kesteren, E.C. Cosman, W.A.J.A. van der Poel, and C.T. Foxon, Phys. Rev B 41 (1990) 5283-5292

[2] S. Permogorov, A. Naumov, C. Gourdon and P. Lavallard, Solid State Commun. 74 (1990) 1057-1061

[3] W.A.J.A. van der Poel, A.L.G.J. Severens, and C.T. Foxon, Opt. Commun. 76 (1990) 116-120

[4] C. Gourdon and P. Lavallard, Phys. Rev. B 46 (1992) 4644-4650

[5] I.L. Aleiner and E.L. Ivchenko, Pis'ma Zh. Eksp. Teor. Fiz. 55 (1992) 662-664 [JETP Letters 55 (1992) 692-695]

E.L. Ivchenko, A.Yu. Kaminski, I.L. Aleiner, submitted to JETP

[6] P.G. Baranov, I.V. Mashkov, N.G.Romanov, P. Lavallard, R.Planel, to be published

[7] E.L. Ivchenko, V.P. Kochereshko, A. Yu Naumov, I.N. Uraltsev and P. Lavallard, Superl. and Microstr. 10 (1991) 497-501

[8] P.Lavallard, C. Gourdon, R. Planel, Superlat. and Microstr. 12 (1992) 321-325 